\title{
The Swedish Heart Failure Registry: a living, ongoing quality assurance and research in heart failure
}

\author{
Gianluigi Savarese ${ }^{a}$, Peter Vasko ${ }^{b}$, Åsa Jonssonc, Magnus Edner ${ }^{a}$, Ulf Dahlström ${ }^{d}$ and Lars H. Lund ${ }^{a}$

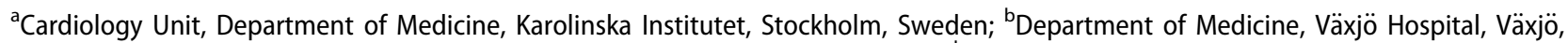 \\ Sweden; 'Department of Medicine, Ryhov County Hospital, Jönköping, Sweden; ${ }^{d}$ Department of Cardiology and Department of Medical and \\ Health Sciences, Linköping University, Linköping, Sweden
}

\begin{abstract}
Heart failure (HF) represents a global pandemic. Although in HF with reduced ejection fraction (HFrEF) randomized controlled trials have provided effective treatments, prognosis still remains poor, with signals of undertreatment. HF with mid-range EF (HFmrEF) has no evidence-based therapy, and its characterization is ongoing. Trials in HF with preserved EF (HFpEF) have failed to provide any effective treatment, but there are several concerns about their design. Thus, current challenges in the HF field are: 1) optimizing the use of existing treatments in HFrEF; 2) developing and proving efficacy of new treatments, and of new use of existing treatments in HFpEF and HFmrEF. Here we describe how registry-based research can improve knowledge addressing the unmet needs in HF, and in particular we focus on the contribution of the Swedish Heart Failure Registry to this field.
\end{abstract}

ARTICLE HISTORY

Received 7 May 2018

Revised 13 June 2018

Accepted 14 June 2018

\section{KEYWORDS}

Heart failure; registry; RiksSvikt; SwedeHF

\section{Introduction}

Heart failure (HF) represents a global pandemic with around 26 million affected worldwide. It is the leading cause of hospitalization in the United States and in Europe and a significant driver of the rising health care costs $(1,2)$. Prognosis remains poor, with $<50 \%$ 4-year survival, similar to the most common cancers, and quality of life is also poor $(3,4)$. In this review we will address contemporary challenges in HF and how registries in general and The Swedish Heart Failure Registry (SwedeHF) in particular may address them.

Around half of the HF population has $\mathrm{HF}$ with reduced ejection fraction (HFrEF; $\mathrm{EF}<40 \%$ ), one-quarter has $\mathrm{HF}$ with mid-range $\mathrm{EF}(\mathrm{HFmrEF} ; \mathrm{EF}=40 \%-49 \%)$, and one-quarter has HF with preserved EF (HFpEF; EF $\geq 50 \%$ ) (5). Trials in HFrEF have yielded several drugs and devices significantly improving survival/morbidity, but prognosis still remains poor and there are signals of treatment under-use; the challenges here include optimizing implementation of existing evidence, or implementation science (6). In HFpEF, trials have not succeeded in demonstrating the efficacy of any tested treatments, but several concerns about study design have been raised. HFmrEF has emerged only recently as an independent entity, and its characterization is ongoing (7). Previously, HFmrEF patients have been enrolled inconsistently in HFpEF or HFrEF trials. Although there are signals that HFrEF treatments may be beneficial also in the HFmrEF population (8-10), ad hoc randomized trials in this subpopulation are missing. The challenges in HFmrEF and HFpEF are primarily innovation science, i.e. developing and proving efficacy of new treatments, and of new use (i.e. for HFmrEF and HFpEF) of existing treatments $(7,8,11)$. Finally, outcomes in acute $\mathrm{HF}$ (AHF) have not improved, and the challenge here, again, is innovation science, new medical and device interventions, as well as new and better use of existing interventions.

\section{The Swedish Heart Failure Registry: an overview}

The Swedish Heart Failure Registry (SwedeHF) (12), or RiksSvikt in Swedish, is a nationwide continuous health quality and research registry founded in 2000 and implemented throughout Sweden in 2003. Up to 31 December 2014, 101,303 registrations from 63,519 unique patients have been included from 70 of a total of 80 hospitals (both inpatients and outpatients) and from 100 of a total of 1000 outpatient primary care clinics in Sweden, with around 10,000 registrations added every year. Coverage of prevalent HF in the inpatient setting is $54 \%$. Coverage of prevalent HF in primary care is much lower, but few patients are seen exclusively in primary care and thus are eventually caught and registered in cardiology or internal medicine departments. Coverage of incident HF is around 10\% (13)-much lower, because the first contact with HF usually occurs at a caregiver that does not report to the registry, such as in the emergency department.

SwedeHF has clinician-judged HF as the only inclusion criterion. EF is not mandatory, but recorded in around $90 \%$ of the registrations, and, thus, distinctions can be made between HFpEF, HFmrEF, and HFrEF patients. Care in 
pediatric departments is the only exclusion criterion. Individual patient consent is not required, but patients are informed of entry into national quality registries and allowed to opt-out.

In SwedeHF approximately 80 variables are entered, at hospital discharge or after outpatient clinic visit, onto a webbased case report form. From these, numerous additional variables are derived by the data capture system, such as sex (derived from universal personal identification number), or subsequently during data management or statistical analysis (such as estimated glomerular filtration rate). The number and complexity of variables were carefully chosen. Indeed, an adequate number of variables needs to be provided in order to enable a detailed characterization and phenotyping of HF population. However, a case report form including too many variables might be excessively time-consuming and thus unpractical to be filled in in routine care. This might reduce feasibility, enrollment, and thus external validity and generalizability of data. Thus, SwedeHF includes creatinine, but also e.g. N-terminal pro-B-type natriuretic peptide (NTproBNP), potassium, and hemoglobin, but not a number of other biomarkers that are used generally in research only; and it includes EF but no additional echo parameters. The Uppsala Clinical Research Center (UCR) manages the database. Thus, data on demographics (age, gender, etc.), clinical characteristics (X-ray, ECG, heart rate, blood pressure, New York Heart Association class), laboratory tests (creatinine, potassium, hemoglobin, NT-proBNP, etc.), comorbidities, and cardiovascular treatments are collected. Furthermore, additional baseline categorical variables can be obtained by linking SwedeHF with other government and disease registries by the personal identification number that all permanent residents in Sweden have regardless of citizenship. In particular, the National Patient Registry (Socialstyrelsen-The National Board of Health and Welfare) provides additional data on comorbidities as ICD-10 codes (e.g. stroke, peripheral artery disease, cancer, renal disease, dialysis, etc.), while socioeconomic data (e.g. level of education, income, employment, etc.) are extracted from Statistics Sweden (Statistiska Centralbyrån), which can also provide age- and gendermatched controls without HF. Coverage of the National Patient Registry approximates, while that of Statistics Sweden is $100 \%$. ICD-10 coding in Sweden has been validated, with a positive predictive value ranging between $85 \%$ and $95 \%$ for most diagnoses; for example, a HF diagnosis has been verified in $86 \%-91 \%$ of cases $(14,15)$. Additional data on treatments can be obtained by linking with the Dispensed Drug Registry from Socialstyrelsen (available since 2005).

Regarding outcomes, vital status and any date of death are provided by the Population Registry (administered by Socialstyrelsen), whereas data on cause-specific hospitalization, new-onset morbidity, and cause of death can be extracted by linking with the National Patient Registry. Vital status is updated monthly, medication adherence continuously, data on cause of death, hospitalization, and new-onset morbidity yearly.
SwedeHF can be also linked with other national disease registries and cohorts. For example, the linking with SCREAM (Stockholm Creatinine Measurement Project), which is a repository of laboratory data of individuals residing or accessing health care in Stockholm County, allows longitudinal assessment of the association between HF therapies and changes in relevant laboratory values (e.g. incidence of hyperkalemia in $\mathrm{HF}$ patients receiving mineralocorticoid receptor antagonists [MRAs]) (16).

Example 1: enrollment in SwedeHF associated with improved outcomes

Enrollment in registries has been assumed to be associated with an improvement in outcome, but this had never been proven until 2017, when an analysis of 231,437 HF patients showed a dramatic reduction in mortality in those registered in both SwedeHF and the National Patient Registry versus those registered only in the latter (13). This observation was in part explained by the different characteristics of patients registered versus those not registered in SwedeHF (e.g. younger age, higher education, less comorbidities), but a major role was played by the better use of HF-specific treatments in patients enrolled in SwedeHF (13).

Example 2: SwedeHF for studying implementation of evidence-based treatments in HFrEF

Even in registries where enrollment is associated with improved outcome linked to better treatments and care there is evidence of suboptimal use of HF-related and more general cardiovascular (CV) therapies (6). Thus, analyses from registries may be determinant to study factors associated with undertreatment and foster implementation of evidencebased therapies.

Data from SwedeHF have been valuable to assess trends in use of HFrEF therapies over time in Sweden, with findings potentially generalizable to or useful for comparison with other European countries and United States. In 5908 patients with New York Heart Association (NYHA) class II-IV, EF $<30 \%$, and HF duration $\geq 6$ months enrolled in SwedeHF between 2003 and 2012, renin-angiotensin system inhibitors (RASi) and beta-blocker use were overall high, $\approx 90 \%$, and constant over time (6). On the other hand, use of cardiac resynchronization therapy (CRT) and implantable cardioverter defibrillator (ICD) was very low, and increased from $2.4 \%$ and $4.0 \%$ in 2003 to only $8.2 \%$ and $10.7 \%$ in 2012 , respectively (6). This study prompted an examination of the quality of HF care in Sweden, and the largest public insurer, Stockholm County Council (SLL), funded the so-called 4D Heart Failure project to improve access to and quality of HF care (http:// viss.nu/Nyheter/Projekt-4D-Hjartsvikt/).

In the same study, use of MRAs was modest, $\approx 53 \%$ in 2003, and decreased over time to $\approx 42 \%$ in 2012 (6). Reasons for MRA under-use were assessed in SwedeHF and included reduced renal function as expected, even in the creatinine clearance $30-59.9 \mathrm{~mL} / \mathrm{min}$ range where MRAs are not contraindicated (17).

Undertreatment in the HF population is not limited to HFspecific therapy. In all patients with HF and concomitant 
atrial fibrillation (AF), oral anticoagulation (OAC) is recommended by guidelines since $\mathrm{CHA}_{2} \mathrm{DS}_{2}$-VASC is $\geq 1(18,19)$. However, data from SwedeHF reported that only $58 \%$ of HF patients with concomitant $\mathrm{AF}$ received $\mathrm{OAC}$, and the underestimation of thrombotic risk and overestimation of bleeding risk were the major determinants of OAC undertreatment (18). Indeed, in our analysis $\mathrm{CHA}_{2} \mathrm{DS}_{2}$-VASC and HAS-BLED scores similarly predicted risk of all-cause mortality/stroke and all-cause mortality/major bleeding, respectively, but HAS-BLED had a considerable higher weight in driving OAC use decision-making compared with $\mathrm{CHA}_{2} \mathrm{DS}_{2}$-VASc (18).

These studies highlight the role of SwedeHF in assessing use of therapies in real-world HF patients and identifying the reasons for potential undertreatment. The data provided support the hypothesis that although developing new treatments is key to improving prognosis, implementing the utilization of existing evidence-based interventions is also critically important.

Example 3: SwedeHF for HF characterization and phenotyping

Several studies from SwedeHF have contributed to the improving understanding of HFpEF and HFmrEF. HFmrEF has been shown to be an intermediate phenotype between HFpEF and HFrEF for most relevant patient characteristics, such as age, gender, body mass index, hypertension, atrial fibrillation, and anemia, but, notably, more similar to HFrEF for prevalent and incident ischemic heart disease $(5,20)$. Regarding prognosis, crude mortality has been reported to be lower in HFmrEF and HFrEF versus HFpEF, but adjusted risk was lower in HFmrEF and HFpEF, with ischemic heart disease portending a higher adjusted risk of death in HFmrEF and $\operatorname{HFrEF}(5,20)$.

SwedeHF data have been compared with data from other international cohorts, providing insights on geographical differences in HF populations $(21,22)$. Indeed, a comparative analysis of SwedeHF versus TaHeF, the Tanzania HF study, has shown Tanzania HF patients to be younger and more commonly female, to have more frequent hypertension and anemia, more advanced HF although with higher EF, and, notably, worse crude but similar adjusted survival (21). Swedish white HF patients have also been compared with those from a Singaporean Asian HF cohort to investigate any differences in the association of QRS duration with EF and outcomes (22). Asian patients were younger, had smaller body size, lower EF, and shorter unadjusted QRS (22). The association between lower EF and longer QRS duration was greater among Asians than whites, with adjusted QRS duration similar in Asians and whites with HFpEF, but longer in Asian versus whites with HFrEF (22). Longer QRS durations was similarly associated with increased risk of mortality/HF hospitalization in both populations (22).

Although in SwedeHF echo and biomarker data are limited, novel methods can be used to improve characterization. Applying machine learning methods to SwedeHF, cluster analysis has identified four different phenotypes significantly differing in outcomes and in response to therapies (23). Additionally, this method has been shown to provide excellent calibration and discrimination for survival, whereas EF has not (23). Thus, applying machine learning to SwedeHF has demonstrated the potential of novel analytic approaches to enhance effectiveness of current therapies that may be translated to a new trial design concept.

Example 4: SwedeHF for identifying potential treatments in $\mathrm{HFpEF}$ and in neglected HFrEF subpopulations

Trials on RASi in HFpEF did not show any efficacy in terms of reduction of primary outcome, but some signals toward benefit were reported (24). Selection bias, underpowering, or high cross-over rates may be potential explanations. Thus, the hypothesis that RASi use may be associated with improved survival was tested in the SwedeHF HFpEF population, where reduced mortality rates were reported in patients treated versus those not treated with RASi in a propensity score matching analysis (25).

Similarly, SwedeHF provided important insights about the use of beta-blockers in HFpEF, where data are sparse and inconclusive. In a propensity score matching analysis, betablockers were associated with a significant reduction in risk of all-cause mortality but not of all-cause mortality or HF hospitalization (26).

Renal insufficiency is common in HF and associated with worse outcome. Patients with renal insufficiency have been excluded from RASi trials in HFrEF, although there is evidence that RASi may be even more beneficial in this subpopulation (27). Thus, in a propensity score matching analysis from SwedeHF, we tested the association between RASi and mortality in HFrEF patients with renal insufficiency defined as creatinine $>221 \mu \mathrm{mol} / \mathrm{L}$ or creatinine clearance $<30 \mathrm{~mL} / \mathrm{min}$, showing a significant improved survival in RASi versus nonRASi users (28).

Although observational data cannot assess efficacy of treatments but only association with outcomes because of unmeasured confounding factors, these important analyses from SwedeHF encourage further testing of these drugs in appropriately powered and designed randomized trials, providing not expensive new interventions but evidence for new use of existing generic inexpensive interventions.

\section{Example 5: SwedeHF for improving trial design}

One of the hypotheses for previous HFpEF trial failures has been wrong patient selection leading to the enrollment of patients who may not have had HF or may have had mild HFpEF, i.e. a poorly enriched population, meaning low risk of $\mathrm{CV}$ events or high risk of non-CV events, that makes testing new HF therapies ineffective or requiring an excessive sample size. NT-proBNP has been used in trials to ensure the presence of HF and enrich for CV events, but the cut-offs for NT-proBNP used to enroll patients have been arbitrary. Thus we investigated in SwedeHF the association between NTproBNP and CV versus non-CV, and our findings led us to conclude that when designing $\mathrm{HF}$ trials it is important to recognize that non-CV events will be higher with higher EF, that potential CV treatment benefits in relation to non-CV events will be lower with higher EF, and that enrolling 
patients with higher NT-proBNP will enrich for CV and also for $\mathrm{CV}$ versus non-CV events but will lead to a more difficult enrollment in terms of numbers of eligible patients (29).

Another potential explanation for HFpEF phase III trials failure could be the inappropriate choice of or interpretation of surrogate endpoints in the preceding phase II trials, meaning e.g. biomarkers whose changes in levels in phase II trials were not associated with changes in prognosis. In SwedeHF we identified a strong association between NT-proBNP levels and risk of all-cause mortality/HF hospitalization regardless of $\mathrm{EF}(29,30)$, and for the first time in an HFpEF/HFmrEF unselected cohort we showed that reduction over time in NTproBNP levels was associated with improved outcome (31). These findings support the use of NT-proBNP as surrogate endpoint in $\mathrm{HFpEF}$ and $\mathrm{HFmrEF}$ phase II trials.

Example 6: a novel paradigm combining randomization as in an RCT with efficiency of a registry-the registry-based randomized controlled trials: SPIRRIT-HFpEF

Registry-based prospective randomized clinical trials (RRCT), with their simplified regulatory, ethics, and consent procedures, and simplified or automated baseline and outcome collection, allow testing novel use of generic drugs or existing interventions in the real-world care setting, with substantially lower costs $(11,32,33)$. SwedeHF has been key to designing and running the Spironolactone Initiation Registry Randomized Interventional Trial in Heart Failure with Preserved Ejection Fraction (SPIRRIT-HFpEF), the first RRCT in HF (11). Briefly, SPIRRIT-HFpEF is a prospective randomized, multicenter, safety/efficacy, parallel assignment, intention-totreat, open-label treatment, phase IV, event-driven interventional trial in $\mathrm{HFpEF}$, testing spironolactone + usual care versus usual care alone in patients with HFpEF. The RRCT platform screens and determines eligibility for both previously and newly entered patients in SwedeHF. Eligible patients who consent are then randomized in the web platform. Baseline data entered into the registry as part of routine care together with randomized assignment are fed into a separate electronic data capture system. Outcomes (death, cause-specific hospitalization, safety, medication adherence and use) are collected by linking SwedeHF with national registries from the Swedish Board of Health and Welfare. Enrollment in SPIRRIT-HFpEF is currently ongoing, and 3200 patients will be randomized by 2020 .

\section{Conclusions}

Over the last 10 years SwedeHF has reported on and improved quality of HF care in Sweden and has provided important research findings that have contributed to substantially improve our understanding and care of HF. Registry-based studies have a major role to identify undertreatment and, thus, implement the use of existing therapies. Notably, data from registries can support trial design directly, as in RRCTs, or indirectly by fostering patients' characterization, the identification of potential treatments and optimal outcomes for trials.

\section{Disclosure statement}

G.S.: related to present work: none. Unrelated to present work: research grants from Merck Sharp \& Dohme, Boehringer Ingelheim, Swedish Heart and Lung Foundation.

P.V.: related to present work: none.

A.J.: related to present work: none.

M.E.: related to present work: none.

U.D.: related to present work: none. Unrelated to present work: research grants to the author's institution from AstraZeneca and speaker's and/or consulting fees from AstraZeneca and Novartis.

L.H.L.: related to present work: none. Unrelated to present work: grants and consulting from Novartis, grants and consulting from Boehringer Ingelheim, consulting from Sanofi, consulting from Vifor Pharma, grants and consulting from AstraZeneca.

\section{Notes on contributors}

Gianluigi Savarese is a cardiologist and assistant professor of cardiology at the Department of Medicine, Karolinska Institutet, Stockholm, Sweden.

Peter Vasko is a cardiologist at the Department of Medicine, Växjö Hospital, Växjö, Sweden. He is chairman of SwedeHF.

Assa Jonsson is a research nurse and $\mathrm{PhD}$ candidate at the Department of Medicine, Division of Cardiology, County hospital Ryhov, Jönköping, Sweden and at Department of Medical and Health Sciences, Linköping University, Linköping, Sweden. She is national coordinator of SwedeHF.

Magnus Edner is a cardiologist and associate professor of cardiology at the Department of Medicine, Karolinska Institutet, Stockholm, Sweden. He is cofounder of SwedeHF.

Ulf Dahlström is a cardiologist and professor of cardiology at the Department of Medical and Health Sciences, Linköping University, Linköping, Sweden. He is cofounder and chairman of research of SwedeHF.

Lars $H$. Lund is a cardiologist and professor of cardiology at the Department of Medicine, Karolinska Institutet, Stockholm, Sweden. He is member of the SwedeHF steering committee for the registry and for research.

\section{Funding}

This study was supported in part by grants to L.H.L.'s institution from the Swedish Research Council [grants 2013-23897-104604-23 and 5232014-2336], the Swedish Heart and Lung Foundation [grants 20120321 and 20150557], and Relypsa Inc. No funding agency had any role in the design and conduct of the study, collection, management, analysis, or interpretation of the data, or in the preparation or approval of the manuscript.

\section{References}

1. Ambrosy AP, Gheorghiade M, Chioncel O, Mentz RJ, Butler J. Global perspectives in hospitalized heart failure: regional and ethnic variation in patient characteristics, management, and outcomes. Curr Heart Fail Rep. 2014;11:416-27. 
2. Savarese G, Lund LH. Global public health burden of heart failure. Card Fail Rev. 2017;3:7-11.

3. Henkel DM, Redfield MM, Weston SA, Gerber Y, Roger VL. Death in heart failure: a community perspective. Circ Heart Fail. 2008;1:91-7.

4. Juenger J, Schellberg D, Kraemer S, Haunstetter A, Zugck $C$, Herzog W, et al. Health related quality of life in patients with congestive heart failure: comparison with other chronic diseases and relation to functional variables. Heart. 2002;87:235-41.

5. Koh AS, Tay WT, Teng THK, Vedin O, Benson L, Dahlstrom U, et al. A comprehensive population-based characterization of heart failure with mid-range ejection fraction. Eur $J$ Heart Fail. 2017;19:1624-34.

6. Thorvaldsen T, Benson L, Dahlstrom U, Edner M, Lund LH. Use of evidence-based therapy and survival in heart failure in Sweden 2003-2012. Eur J Heart Fail. 2016;18:503-11.

7. Lund LH. Heart Failure With "Mid-Range" Ejection Fraction-New Opportunities. J Card Fail. 2016;22:769-71.

8. Lund LH, Claggett B, Liu J, Lam CS, Jhund PS, Rosano GM, et al. Heart failure with mid-range ejection fraction in CHARM: characteristics, outcomes and effect of candesartan across the entire ejection fraction spectrum. Eur J Heart Fail. 2018 Feb 12; doi: 10.1002/ejhf.1149. [Epub ahead of print]

9. Solomon SD, Claggett B, Desai AS, Packer M, Zile M, Swedberg K, et al. Influence of ejection fraction on outcomes and efficacy of sacubitril/valsartan (LCZ696) in heart failure with reduced ejection fraction: the Prospective Comparison of ARNI with ACEI to Determine Impact on Global Mortality and Morbidity in Heart Failure (PARADIGM-HF) Trial. Circ Heart Fail. 2016;9:e002744.

10. Solomon SD, Claggett B, Lewis EF, Desai A, Anand I, Sweitzer NK, et al. Influence of ejection fraction on outcomes and efficacy of spironolactone in patients with heart failure with preserved ejection fraction. Eur Heart J. 2016;37:455-62.

11. Lund LH, Oldgren J, James S. Registry-based pragmatic trials in heart failure: current experience and future directions. Curr Heart Fail Rep. 2017;14:59-70.

12. Jonsson $A$, Edner $M$, Alehagen $U$, Dahlstrom U. Heart failure registry: a valuable tool for improving the management of patients with heart failure. Eur J Heart Fail. 2010;12:25-31.

13. Lund LH, Carrero JJ, Farahmand B, Henriksson KM, Jonsson Å, Jernberg $\mathrm{T}$, et al. Association between enrolment in a heart failure quality registry and subsequent mortality-a nationwide cohort study. Eur J Heart Fail. 2017;19:1107-16.

14. Ludvigsson JF, Andersson E, Ekbom A, Feychting $M$, Kim JL, Reuterwall $C$, et al. External review and validation of the Swedish national inpatient register. BMC Public Health. 2011;11:450.

15. Ingelsson E, Arnlov J, Sundstrom J, Lind L. The validity of a diagnosis of heart failure in a hospital discharge register. Eur J Heart Fail. 2005;7:787-91.

16. Trevisan $M$, de Deco $P, X u H$, Evans $M$, Lindholm B, Bellocco R, et al. Incidence, predictors and clinical management of hyperkalaemia in new users of mineralocorticoid receptor antagonists. Eur J Heart Fail. 2018 Apr 18; doi: 10.1002/ejhf.1199. [Epub ahead of print]

17. Savarese G, Carrero JJ, Pitt B, Anker SD, Rosano GMC, Dahlström $\mathrm{U}$, et al. Factors associated with underuse of mineralocorticoid receptor antagonists in heart failure with reduced ejection fraction: an analysis of 11215 patients from the Swedish Heart Failure Registry. Eur J Heart Fail. 2018 Mar 26; doi: 10.1002/ ejhf.1182. [Epub ahead of print]

18. Savarese G, Sartipy U, Friberg L, Dahlstrom U, Lund LH. Reasons for and consequences of oral anticoagulant underuse in atrial fibrillation with heart failure. Heart. 2018;104:1093-100.

19. Kirchhof $\mathrm{P}$, Benussi $\mathrm{S}$, Kotecha D, Ahlsson A, Atar D, Casadei B, et al. 2016 ESC Guidelines for the management of atrial fibrillation developed in collaboration with EACTS: The Task Force for the management of atrial fibrillation of the European Society of Cardiology (ESC) Developed with the special contribution of the European Heart Rhythm Association (EHRA) of the ESC Endorsed by the European Stroke Organisation (ESO). Eur Heart J. 2016;37:2893-962.

20. Vedin O, Lam CSP, Koh AS, Benson L, Teng THK, Tay WT, et al. Significance of ischemic heart disease in patients with heart failure and preserved, midrange, and reduced ejection fraction: a nationwide cohort study. Circ Heart Fail. 2017;10. pii: e003875.

21. Makubi A, Hage C, Sartipy U, Lwakatare J, Janabi M, Kisenge P, et al. Heart failure in Tanzania and Sweden: comparative characterization and prognosis in the Tanzania Heart Failure (TaHeF) study and the Swedish Heart Failure Registry (SwedeHF). Int J Cardiol. 2016;220:750-8.

22. Gijsberts CM, Benson L, Dahlström U, Sim D, Yeo DP, Ong HY, et al. Ethnic differences in the association of QRS duration with ejection fraction and outcome in heart failure. Heart. 2016;102:1464-71.

23. Ahmad T, Lund LH, Rao P, Ghosh R, Warier P, Vaccaro B, et al. Machine learning methods improve prognostication, identify clinically distinct phenotypes, and detect heterogeneity in response to therapy in a large cohort of heart failure patients. J Am Heart Assoc. 2018;7. pii: e008081.

24. Yusuf S, Pfeffer MA, Swedberg K, Granger CB, Held P, McMurray $\mathrm{JJ}$, et al. Effects of candesartan in patients with chronic heart failure and preserved left-ventricular ejection fraction: the CHARMPreserved Trial. Lancet. 2003;362:777-81.

25. Lund LH, Benson L, Dahlstrom U, Edner M. Association between use of renin-angiotensin system antagonists and mortality in patients with heart failure and preserved ejection fraction. JAMA. 2012;308:2108-17.

26. Lund LH, Benson L, Dahlstrom U, Edner M, Friberg L. Association between use of beta-blockers and outcomes in patients with heart failure and preserved ejection fraction. JAMA. 2014;312:2008-18.

27. Brenner BM, Cooper ME, de Zeeuw D, Keane WF, Mitch WE, Parving $\mathrm{HH}$, et al. Effects of losartan on renal and cardiovascular outcomes in patients with type 2 diabetes and nephropathy. $\mathrm{N}$ Engl J Med. 2001;345:861-9.

28. Edner M, Benson L, Dahlstrom U, Lund LH. Association between renin-angiotensin system antagonist use and mortality in heart failure with severe renal insufficiency: a prospective propensity score-matched cohort study. Eur Heart J. 2015;36:2318-26.

29. Savarese G, Orsini N, Hage C, Vedin O, Cosentino F, Rosano GMC et al. Utilizing NT-proBNP for eligibility and enrichment in trials in HFpEF, HFmrEF, and HFrEF. JACC Heart Fail. 2018;6:246-56.

30. Savarese G, Orsini N, Hage C, Dahlström U, Vedin O, Rosano GMC, et al. Associations with and prognostic and discriminatory role of $\mathrm{N}$-terminal pro-B-type natriuretic peptide in heart failure with preserved vs. mid-range vs. reduced ejection fraction. J Card Fail. 2018;24:365-74.

31. Savarese G, Hage C, Orsini N, Dahlström U, Perrone-Filardi P, Rosano GM, et al. Reductions in N-terminal pro-brain natriuretic peptide levels are associated with lower mortality and heart failure hospitalization rates in patients with heart failure with midrange and preserved ejection fraction. Circ Heart Fail. 2016;9. pii: e003105.

32. Fröbert O, Lagerqvist B, Olivecrona GK, Omerovic E, Gudnason T, Maeng $M$, et al. Thrombus aspiration during ST-segment elevation myocardial infarction. N Engl J Med. 2013;369:1587-97.

33. James S, Rao SV, Granger CB. Registry-based randomized clinical trials-a new clinical trial paradigm. Nat Rev Cardiol. 2015;12: 312-16. 